\title{
AVALIAÇÃO DE MODELO DE EXTRAÇÃO DA ÁGUA DO SOLO POR SISTEMAS RADICULARES DIVIDIDOS ENTRE CAMADAS DE SOLO COM PROPRIEDADES HIDRÁULICAS DISTINTAS ${ }^{(1)}$
}

\author{
Marlon Gomes da Rocha ${ }^{(2)}$, Leandro Neves Faria ${ }^{(2)}$, \\ Derblai Casaroli $^{(3)}$ \& Quirijn de Jong Van Lier ${ }^{(4)}$
}

\begin{abstract}
RESUMO
A avaliação da capacidade de raízes de plantas em extrair água do solo é de grande importância na modelagem da taxa de transpiração e, para entender o crescimento e rendimento vegetal e o balanço de água e de solutos no solo. Para testar um modelo de extração radicular macroscópico baseado no processo em escala microscópica, descreveram-se os resultados de um experimento com plantas cujo sistema radicular foi dividido entre camadas de solo com propriedades hidráulicas contrastantes. Um experimento de lisímetro dividido com plantas de sorgo foi realizado em Piracicaba-SP. Quatro lisímetros com dois compartimentos separados fisicamente (split-pot) foram construídos e preenchidos com material de dois tipos de solo de diferentes classes texturais (um solo de textura média - AR e outro de textura argilosa - AG). Durante um mês e meio foi imposto um regime hídrico, alternando a irrigação entre os compartimentos. O teor de água nos compartimentos dos lisímetros foi monitorado com TDR e tensiômetros. $\mathrm{O}$ material dos dois solos foi analisado conforme método-padrão quanto às suas propriedades de retenção e condução da água. A densidade radicular foi determinada por pesagem no fim do experimento, tendo ficado em torno de duas vezes maior no solo AR do que no AG. Observou-se que a extração de água ocorreu preferencialmente do compartimento do lisímetro com maior potencial de fluxo matricial. Em certas ocasiões houve transferência de água do lado de maior para o de menor potencial de fluxo matricial, com a liberação da água ao solo pelo sistema radicular (hydraulic lift). Para compensar o efeito da heterogeneidade da distribuição radicular e da atividade radicular, incluiu-se, no modelo, um fator empírico $f$ de correção. O modelo testado descreveu bem 80 \% das observações com a utilização de valores de $f$ de 0,01506 e 0,003713 , para os solos AR e AG, respectivamente. O modelo simulou a liberação de água ao solo mais frequentemente do que ela ocorreu no
\end{abstract}

\footnotetext{
(1) Recebido para publicação em abril de 2009 e aprovado em junho de 2010.

(2) Mestrando da PPG em Física do Ambiente Agrícola, Escola Superior de Agricultura "Luiz de Queiroz" - ESALQ/USP. Caixa Postal 9, Av. Pádua Dias 11, CEP 13418-900 Piracicaba (SP). E-mail: agrolon@gmail.com; leandronfaria@gmail.com

(3) Professor do Centro Universitário de Patos de Minas - UNIPAM. Rua Major Gote 808, CEP 38702-054 Patos de Minas (MG). E-mail derblai@unipam.edu.br

(4) Professor do Departamento de Engenharia de Biossistemas, ESALQ/USP. E-mail: qdjvlier@esalq.usp.br
} 
experimento. Esse fato pode indicar que a resistência interna do sistema radicular, não contabilizada pelo modelo, pode ter papel importante nas relações hídricas na rizosfera.

Termos de indexação: modelagem, deficiência hídrica, densidade radicular, transpiração.

\title{
SUMMARY: EVALUATION OF A ROOT-SOIL WATER EXTRACTION MODEL BY ROOT SYSTEMS DIVIDED OVER SOIL LAYERS WITH DISTINCT HYDRAULIC PROPERTIES
}

\begin{abstract}
Evaluating plant root capacity in extrating water from the soil is important for transpiration modeling and to understand crop growth and yield and soil water and nutrient balance. Aiming to test a macroscopic root water extraction model based on the microscopic process description, an experiment was described in which the root system of plants penetrated different soil layers with contrasting hydraulic properties. Four lysimeters containing two physically divided compartments were built and filled with material of two soils with different texture (a medium textured soil - AR and a clayey soil - AG). During a month and a half a water regime was imposed alternating the irrigation among the compartments. The soil water content in the compartments was measured with TDR and tensiometers. Soil hydraulic properties - retention and conductivity - were analyzed by standard methods. Root density was determined by weighing at the end of the experiment, resulting in values twice as high in AR than in AG soil. It was observed that water extraction occurred preferentially from the lysimeter compartments with the highest matric flux potential. Occasionally, water transfer from the compartment with higher matric flux potential to the lower one was observed, releasing water from root to soil (hydraulic lift). To compensate for the effect of heterogeneity of root distribution and root activity and soil-root contact, an empirical factor $\mathrm{f}$ was added to the model. Its value was determined by a numerical fitting procedure aiming at the highest correlation between model and observation in the four lysimeters. The model described $80 \%$ of the observations satisfactorily by using these f values, which were 0.01506 and 0.003713 , respectively, for AR and AG. Model predictions indicated a much more frequent water release from roots to soil than observed in the experiment. This may suggest internal root resistance, not considered by the model, may play an important role in root-water relations.
\end{abstract}

Index terms: Modeling, water stress, root density, transpiration.

\section{INTRODUÇÃO}

A estimativa da taxa de transpiração da vegetação é imperativa em modelos hidrológicos e meteorológicos. A transpiração da vegetação ocorre à taxa potencial $T_{p}$ em condições hídricas do solo ditas não limitantes (van den Berg \& Driessen, 2002). O valor de $T_{p}$ é determinado apenas pelas condições atmosféricas e fatores de geometria e resistência do dossel. Sob condições chamadas "limitantes", as plantas fecham os estômatos, aumentando a resistência estomática e reduzindo a taxa de transpiração e fotossintética e o acúmulo de fitomassa (Jong van Lier et al., 2008). Pela correlação que existe entre a taxa de transpiração e a de fotossíntese, quanto maior a acurácia na estimativa da taxa de transpiração, melhor é o desempenho dos modelos de crescimento vegetal e de rendimento agrícola.
A descrição de extração de água do solo por raízes de plantas é uma ferramenta importante para a modelagem da taxa de transpiração e, consequentemente, do crescimento e rendimento vegetal e do balanço de água e de solutos no solo. Modelos microscópicos analíticos (Gardner, 1960; Cowan, 1965) e numéricos (Jong van Lier et al., 2006) descrevem o fluxo radial em direção a raízes individuais, enquanto modelos macroscópicos (Gardner, 1964; Molz \& Remson, 1970; Molz, 1981; Clausnitzer \& Hopmans, 1994; Jong van Lier et al., 2008) consideram o sistema radicular como um todo, desconsiderando a geometria e extração da água do solo das raízes individuais.

Para a estimativa da taxa de transpiração $T_{\alpha}$ em função das condições hidráulicas no solo, geralmente são empregadas funções de redução empíricas. Essas funções reconhecem uma faixa de condições em que $T_{a}=T_{p}$, a fase de taxa constante. Numa faixa de 
condições mais secas do que um determinado valor crítico, supõe-se que $T_{a}<T_{p}$ e que $T_{a}$ diminui com a redução do teor de água. Por essa razão, a faixa em que $T_{a}<T_{p}$ é também chamada de fase de taxa decrescente (Kozak et al., 2005). Entre as funções empíricas de redução mais utilizadas, na fase de taxa decrescente, a de Doorenbos \& Kassam (1986) supõe uma relação linear entre o teor de água $\theta$ e a transpiração relativa $T_{r}=T_{a} / T_{p}$, enquanto a de Feddes et al. (1978) supõe linearidade entre $T_{r}$ e o potencial matricial $h$.

No caso de um sistema radicular que se estende ao longo de mais do que uma camada do solo, para estimativa da transpiração, os modelos macroscópicos mais simples utilizam médias das propriedades do solo e do sistema radicular, enquanto os mais complexos simulam a extração da água por camada de solo. Utilizam-se as funções de redução mencionadas anteriormente para estimar a redução de transpiração, como a média ponderada dos fatores de redução de cada camada. Assim, se ao menos uma das camadas de solo encontra-se com condições hidráulicas limitantes, o modelo estima uma transpiração reduzida. Dessa maneira, não se prevê a possibilidade da compensação, isto é, de poder compensar a reduzida extração das camadas mais secas com uma extração mais intensa das camadas mais úmidas. Descrições empíricas dessa compensação foram incluídas nos modelos CERES (Ritchie, 1985) e EPIC (Williams, 1995), como discutido por Li et al. (2001a). Outros exemplos de sua aplicação são os modelos de Lai \& Katul (2000) e Li et al. (2001b), que propuseram mecanismos empíricos compensatórios.

Com base em um modelo microscópico de extração radicular, Jong van Lier et al. (2008) propuseram um protocolo de cálculo para modelar a extração de água por um sistema radicular, levando em consideração as características hidráulicas das camadas, sua densidade radicular e seu teor de água. $\mathrm{O}$ método de cálculo inclui uma descrição física do processo de compensação, que foi incorporado no modelo hidrológico SWAP (Kroes \& van Dam, 2003) e mostrou-se promissor quando comparado com dados de experimentos de longa duração.

Há, no entanto, a necessidade de uma comprovação mais rigorosa de funcionalidade do modelo sob condições de contraste entre as camadas enraizadas. Uma forma experimental de impor essas condições é pela utilização de um lisímetro dividido (split-pot): um recipiente especial em que o sistema radicular de uma planta é dividido em duas partes, separadas fisicamente e monitoradas. Método semelhante foi empregado por Lascano \& van Bavel (1986), Kosola \& Eissenstat (1994), Hussain et al. (1999) e Espeleta et al. (2004).

Com o objetivo de testar o modelo de extração radicular de Jong van Lier et al. (2008) e, assim, contribuir com a avaliação da taxa de transpiração de plantas cultivadas, no presente trabalho descrevem-se os resultados de um experimento com plantas cujo sistema radicular foi dividido entre camadas de solo com propriedades hidráulicas contrastantes, sob combinações diversas de teores de água.

\section{MATERIAL E MÉTODOS}

O trabalho foi realizado em quatro lisímetros divididos, que foram cultivados com plantas de sorgo (Sorghum bicolor (L.) Moench), em casa de vegetação localizada na Escola Superior de Agricultura "Luiz de Queiroz" da Universidade de São Paulo (ESALQ/ USP), em Piracicaba - SP, no período de março a junho de 2008.

Os lisímetros, com dimensões internas de $0,5 \times 0,3 \times 0,4 \mathrm{~m}$, foram confeccionados em metal, possuíam drenagem livre e foram divididos longitudinalmente em dois compartimentos (Figura 1).

Em cada lisímetro, um dos dois compartimentos foi preenchido com material de um Argissolo VermelhoAmarelo distrófico típico textura média (AR), com teores de areia, silte e argila de 0,76, 0,04 e 0,20 $\mathrm{kg} \mathrm{kg}^{-1}$, respectivamente. O outro compartimento foi preenchido com material de um Nitossolo Vermelho eutroférrico típico textura argilosa (AG), com teores de areia, silte e argila de 0,39, 0,08 e $0,53 \mathrm{~kg} \mathrm{~kg}^{-1}$, respectivamente, conforme a classificação da Embrapa (1999). O material de ambos os solos foi coletado no campus "Luiz de Queiroz" da USP, Piracicaba.

O preenchimento dos lisímetros com o material do solo previamente seco e peneirado foi feito objetivandose estabelecer as densidades de 1.400 e $1.200 \mathrm{~kg} \mathrm{~m}^{-3}$ para os solos AR e AG, respectivamente, aproximadamente iguais às suas densidades naturais de campo. Guias de onda de TDR foram inseridos em ambos os compartimentos dos lisímetros nas profundidades de $0,05-0,10,0,15-0,20$ e $0,25-0,30 \mathrm{~m}$. A seguir, o

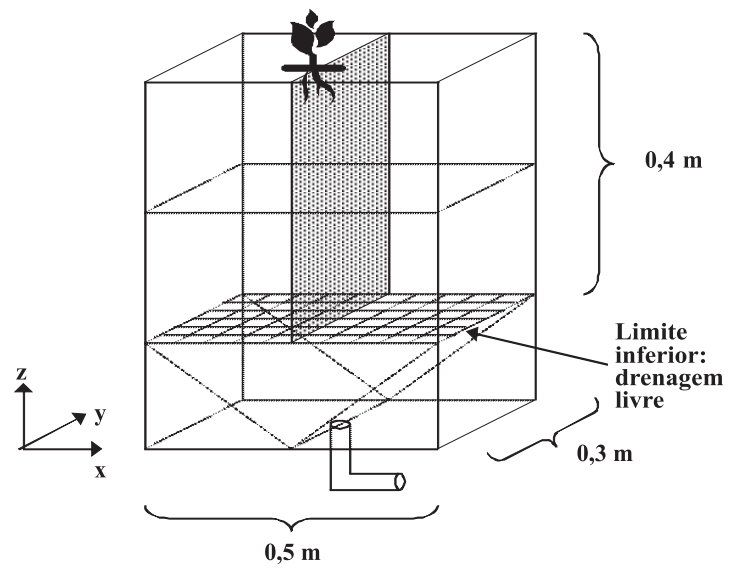

Figura 1. Desenho esquemático de um lisímetro dividido utilizado no experimento, mostrando dimensões e divisão interna. 
lisímetro foi umedecido e deixado por uma semana para assentamento do material de solo. Posteriormente, um tensiômetro foi instalado na profundidade de 5-10 cm para monitorar as condições hídricas e indicar a necessidade de irrigação.

O material de solo de cada compartimento do lisímetro foi adubado segundo a análise química $\mathrm{e}$ seguindo as normas de interpretação do Boletim Técnico 100 (Raij et al., 1997), para a cultura em questão.

Sorgo (Sorghum bicolor (L.) Moench, cultivar Catissorgo LS 6-8), foi semeado em vasos em 25 de fevereiro de 2008. Vinte dias após a germinação, duas plantas foram transplantadas para cada lisímetro, de maneira que metade do seu sistema radicular ficasse em cada compartimento (Figura 2).

A superfície do solo foi coberta com plástico e uma camada de aproximadamente $2 \mathrm{~cm}$ de areia grossa, com o objetivo de minimizar a perda de água por evaporação nos lisímetros. As plantas foram irrigadas manualmente, mantendo-se um teor de água adequado para seu desenvolvimento com um potencial matricial em torno de $-2 \mathrm{~m}$, até atingirem o estádio reprodutivo.

Em 8 de maio de 2008, 72 dias após a semeadura, iniciaram-se os tratamentos de irrigação e a coleta de dados para caracterizar o estado hídrico do solo, realizados simultaneamente por meio de leituras do TDR e do tensiômetro, três vezes ao dia, às 8, 13 e $18 \mathrm{~h}$, aproximadamente.

O tratamento de irrigação foi alternado, distinguindo-se quatro fases: fase I ( 12 a 23 de maio) - irrigação do solo AR e secagem do compartimento com solo AG; fase II (23 de maio a 6 de junho) - sem irrigação e secagem dos dois compartimentos; fase III (6 a 13 de junho) - irrigação do compartimento contendo o solo AG e secagem do solo AR; e fase IV (13 a 23 de junho) - sem irrigação e secagem dos dois compartimentos.

A irrigação do solo AR ou AG nas fases I e III foi realizada sempre que o tensiômetro do respectivo compartimento indicava um potencial matricial de
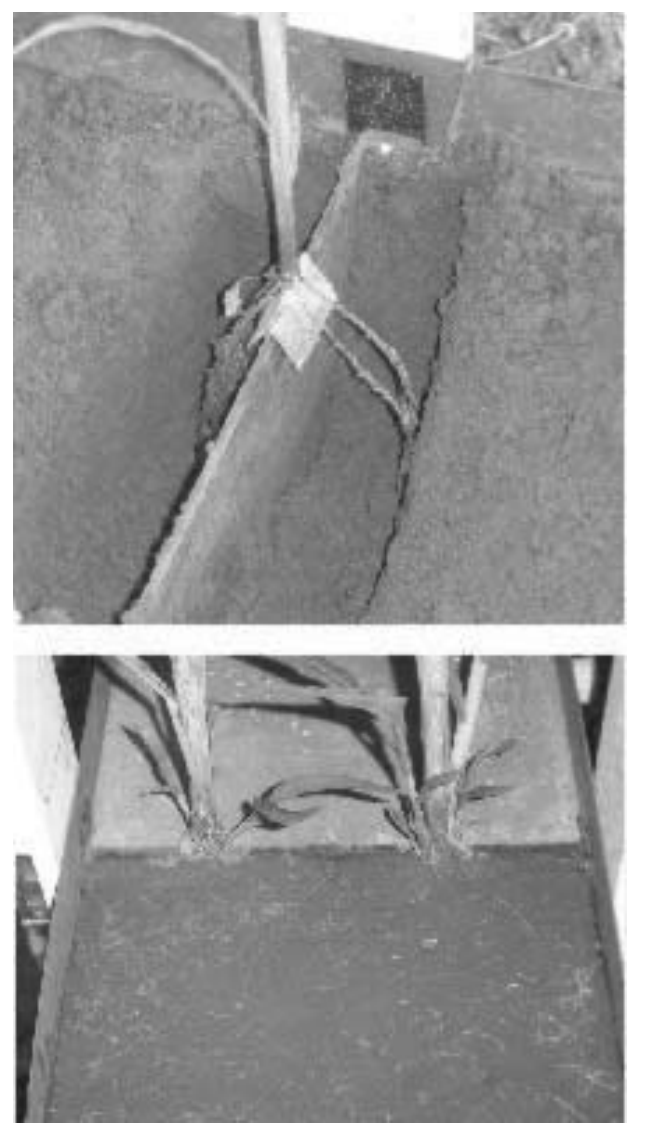

Figura 2. Posição de uma planta e divisão de seu sistema radicular nos compartimentos de um lisímetro no momento do transplante (esquerda) e disposição das plantas já na fase adulta (direita).

$-2 \mathrm{~m}$. As lâminas aplicadas estão representadas no quadro 1 para os quatro lisímetros.

Para fins de quantificação do sistema radicular, ao final do experimento os lisímetros foram desmontados e amostras de solo foram coletadas por camada. As raízes foram separadas do solo cuidadosamente por lavagem com água, sobre uma

Quadro 1. Lâminas de água ( $\mathrm{mm}$ ) aplicadas nos compartimentos com material do solo AR e AG para os lisímetros 1, 2, 3 e 4 ao longo do período experimental

\begin{tabular}{|c|c|c|c|c|c|c|c|c|c|}
\hline \multirow{2}{*}{ Fase } & \multirow{2}{*}{ dia/mês } & \multicolumn{4}{|c|}{ AR } & \multicolumn{4}{|c|}{$\mathrm{AG}$} \\
\hline & & 1 & 2 & 3 & 4 & 1 & 2 & 3 & 4 \\
\hline \multirow[t]{6}{*}{ I } & $12 / 05$ & 13,3 & & & & & & & \\
\hline & $13 / 05$ & & & 13,3 & & & & & \\
\hline & $18 / 05$ & & & & 13,3 & & & & \\
\hline & $19 / 05$ & 13,3 & & 13,3 & & & & & \\
\hline & $22 / 05$ & 20,0 & & & & & & & \\
\hline & $23 / 05$ & & 20,0 & 20,0 & 20,0 & & & & \\
\hline \multirow[t]{4}{*}{ III } & $06 / 06$ & & & & & 26,7 & 26,7 & 26,7 & 26,7 \\
\hline & $07 / 06$ & & & & & 6,7 & 6,7 & 6,7 & 6,7 \\
\hline & $12 / 06$ & & & & & 13,3 & 13,3 & & 13,3 \\
\hline & $13 / 06$ & & & & & & & 13,3 & \\
\hline
\end{tabular}


peneira com malha de $1 \mathrm{~mm}$. Esse método foi discutido em Amato \& Pardo (1994), que, para o tamanho da malha utilizado, encontraram perdas de massa de $25 \%$, enquanto as perdas de comprimento foram da ordem de $66 \%$. As raízes separadas foram levadas para uma estufa ventilada a $65^{\circ} \mathrm{C}$ por um período de $48 \mathrm{~h}$, para posterior determinação da matéria seca. Imagens das amostras de raízes foram digitalizadas e utilizadas para obtenção de um valor médio do raio das raízes $r_{0}$. Com base na massa da matéria seca, densidade e no raio das raízes, calculouse o comprimento radicular $\mathrm{L}(\mathrm{m})$ :

$$
L=\frac{m}{d r_{0}^{2} \pi}
$$

em que $m(\mathrm{~kg})$ é a massa da matéria seca; $d\left(\mathrm{~kg} \mathrm{~m}^{-3}\right)$, a massa específica do tecido radicular; e $r_{0}(\mathrm{~m})$, o raio médio das raízes. Encontrou-se o valor de 0,0003 m para $r_{0}$. Para a massa específica do tecido radicular $d$ utilizou-se $500 \mathrm{~kg} \mathrm{~m}^{-3}$, conforme encontrado por Craine \& Lee (2003). Possíveis erros introduzidos na estimativa de $L$ pela equação 1 são discutidos em van Noordwijk \& Brouwer (1991), que apontam a grande variação de $r_{0}$, naturalmente presente, bem como variações na massa específica radicular $d$ devido à presença de ar no tecido radicular. A densidade radicular $\left(R, \mathrm{~m} \mathrm{~m}^{-3}\right)$ foi determinada pelo quociente entre comprimento total de raízes $(L)$ e volume de solo do compartimento do lisímetro $\left(0,0277 \mathrm{~m}^{3}\right)$. Em função da geometria do sistema (Jong van Lier et al., 2006), a relação entre $R$ e a meia distância média entre as raízes $\left(r_{m}, \mathrm{~m}\right)$ pode ser calculada pela equação:

$$
r_{m}=\sqrt{\frac{1}{\pi R}}
$$

A calibração do valor da constante dielétrica $\varepsilon$ lido pelo TDR e do teor de água no solo foi realizada em material dos mesmos solos, acomodado em uma caixa de isopor de mesma densidade que dos lisímetros. Uma leitura da constante dielétrica foi obtida diariamente, seguida de pesagem da caixa e adição de água. O resultado das pesagens permitiu obter o teor de água do solo na caixa. Com os resultados, obtiveram-se os parâmetros $a$ e $b$ da equação de calibração:

$$
\theta=a \varepsilon+b
$$

Para caracterização hidráulica dos solos, foram confeccionadas seis amostras por solo, em anéis volumétricos de 4,6 cm de diâmetro interno e $3,0 \mathrm{~cm}$ de altura, com densidade igual à dos lisímetros. As amostras foram saturadas por um período de $24 \mathrm{~h}$ em uma bandeja com água; e, em seguida, equilibradas aos potenciais matriciais $h$ de $-0,1,-0,2 \mathrm{e}-0,4 \mathrm{~m}$ em mesas de tensão de $-1,-3,-5,-10,-50$ e $-150 \mathrm{~m}$, respectivamente, em câmara de pressão de Richards. Após atingirem o equilíbrio hidráulico, as amostras foram pesadas, determinando-se seu teor de água $(\theta)$. Aos pares de dados $(\theta, h)$ foi ajustada a equação descrita por van Genuchten (1980):

$$
\Theta=\left[1+|\alpha h|^{n}\right]^{-m}
$$

em que $\Theta=\left(\theta-\theta_{r}\right) /\left(\theta_{s}-\theta_{r}\right), \theta_{s}$ e $\theta_{r}$ são os teores de água residual e de saturação, respectivamente, e $\alpha\left(\mathrm{m}^{-1}\right)$, $m$ e $n$ são parâmetros empíricos da equação.

A determinação da condutividade hidráulica $K$ $\left(\mathrm{m} \mathrm{d}^{-1}\right)$ em função do potencial matricial $h(\mathrm{~m})$ foi realizada em amostras sob evaporação no laboratório de física do solo de ESG/WUR - Holanda, seguindo o método de Wind (1968). Cilindros de $103 \mathrm{~mm}$ de diâmetro e $80 \mathrm{~mm}$ de altura foram preenchidos com material dos solos na referida densidade e colocados em água para saturar durante $24 \mathrm{~h}$. O potencial matricial da água foi medido por um conjunto de quatro microtensiômetros, inseridos na amostra por quatro furos laterais de $4 \mathrm{~mm}$ de diâmetro, a 10, 30, 50 e $70 \mathrm{~mm}$ da base da amostra. Os microtensiômetros foram ligados a um transdutor de pressão por meio de um tubo de náilon. O cilindro de solo foi colocado sobre uma placa de plástico rígido e vedado com fita, para evitar vazamento. O conjunto anel-amostratensiômetros foi colocado sobre uma balança. Os transdutores de pressão e a balança foram conectados a um multiplexador associado a um microcomputador. As leituras dos sensores e da balança foram registradas a cada 60 min durante uma semana, enquanto ocorria a evaporação. Após a parte experimental de laboratório, os dados foram processados por modelagem inversa (van Dam et al., 1994), obtendo-se pares de dados $K$ - $h$. Aos valores obtidos foi ajustada a equação:

$$
\begin{gathered}
K=K_{s} ; \text { para }|h| \leq\left|h_{b}\right| \\
K=K_{s}\left(\frac{\left|h_{b}\right|}{|h|}\right)^{b} ; \text { para }|h|>\left|h_{b}\right|
\end{gathered}
$$

em que $K_{s}\left(\mathrm{~m} \mathrm{~d}^{-1}\right)$ é a condutividade hidráulica do solo saturado; $h_{b}(\mathrm{~m})$, o potencial matricial de entrada de ar; e $b$, um parâmetro empírico, os quais foram obtidos por ajuste não linear.

A propriedade hidráulica potencial de fluxo matricial $\left(M, \mathrm{~m}^{2} \mathrm{~d}^{-1}\right)$, também chamada de potencial de Kirchhoff (Gardner, 1958; Raats, 1970), é utilizada no modelo de Jong van Lier et al. (2008) para descrição da extração radicular e da transpiração da planta. Ela é definida como a condutividade hidráulica $\left(K(h), \mathrm{m} \mathrm{d}^{-1}\right)$ integrada num intervalo de potencial matricial. Considerando o potencial matricial correspondente ao ponto de murcha permanente $\left(h_{p m p}\right.$, $\mathrm{m})$ como limite inferior da integração para obtenção de $M$, tem-se:

$$
M=\int_{h_{p m p}}^{h} K(h) d h
$$

No presente estudo adotou-se $h_{p m p}=-150 \mathrm{~m}$.

Escrevendo a equação de Darcy em termos de $M$, verifica-se que: 


$$
q=-K \frac{d H}{d x}=-K\left[\frac{d h}{d x}+\frac{d z}{d x}+\frac{d p}{d x}\right]=-\left[\frac{d M}{d x}+K \frac{d z}{d x}+K \frac{d p}{d x}\right]
$$

em que $q\left(\mathrm{~m} \mathrm{~d}^{-1}\right)$ é a densidade de fluxo de água; $H$ (m), o potencial hidráulico total; x (m), a distância; $z$ (m), a coordenada vertical; $p(\mathrm{~m})$, o somatório dos demais potenciais da água no sistema, além do matricial e gravitacional. No caso de movimento horizontal ou ao longo de pequenas distâncias com gradientes de potencial matricial relativamente altos, como no caso da extração radicular (eJong van Lier et al., 2006), os termos $K \frac{d z}{d x}$ e $K \frac{d p}{d x}$ podem ser desprezados e a equação 7 é simplificada para:

$$
q=-\frac{d M}{d x}
$$

o que demonstra a ligação direta entre $M$ e o movimento de água no solo no caso de ausência de gradientes de potencial significativos, além do matricial.

Substituindo a equação 5 na equação 6 e resolvendo a integral, obtém-se a seguinte expressão para M, utilizada no presente trabalho:

$$
\begin{gathered}
M=\frac{K_{s}\left|h_{b}\right|^{b}}{b-1}\left[|h|^{1-b}-\left|h_{w}\right|^{1-b}\right] \text {; para } \quad|h| \geq\left|h_{b}\right| \\
M=\frac{K_{s}\left|h_{b}\right|^{b}}{b-1}\left[\left|h_{b}\right|^{1-b}-\left|h_{w}\right|^{1-b}\right]+\left[\left|h_{b}\right|-|h|\right] K_{s} ;
\end{gathered}
$$

para $|h|<\left|h_{b}\right|$.

Pelo modelo de Jong van Lier et al. (2008), a extração radicular $S\left(\mathrm{~m}^{3} \mathrm{~m}^{-3} \mathrm{~d}^{-1}\right)$ no compartimento é estimada por:

$$
S=\rho\left(\bar{M}-M_{0}\right)
$$

em que $\bar{M}\left(\mathrm{~m}^{2} \mathrm{~d}^{-1}\right)$ é o potencial de fluxo matricial correspondente ao teor médio de água no compartimento do lisímetro; $M_{0}\left(\mathrm{~m}^{2} \mathrm{~d}^{-1}\right)$, o potencial de fluxo matricial na superfície radicular, considerado igual em todas as raízes e em ambos os compartimentos; e $\rho\left(\mathrm{m}^{-2}\right)$, um coeficiente que determina a dependência entre o potencial de fluxo matricial e a extração de água pelas raízes, definido como:

$$
\rho=\frac{4}{r_{0}{ }^{2}-a^{2} r_{m}{ }^{2}+2\left(r_{m}{ }^{2}+r_{0}{ }^{2}\right) \ln \frac{a r_{m}}{r_{0}}}
$$

Na equação 11, $r_{m}$ foi calculado pela equação 2 para o respectivo compartimento, e o valor de $a$ foi numericamente determinado em 0,53 (Jong van Lier et al., 2006).

Pela equação 10, demonstra-se que o potencial de fluxo matricial na superfície radicular $\left(M_{0}\right)$ pode ser estimado por:

$$
M_{0}=\frac{\rho_{A R} \bar{M}_{A R}+\rho_{A G} \bar{M}_{A G}-\bar{S}_{e}}{\rho_{A R}+\rho_{A G}}
$$

em que os subscritos $A R$ e $A G$ representam os compartimentos do lisímetro com respectivo solo, e $\bar{S}$ $\left(\mathrm{m}^{3} \mathrm{~m}^{-3} \mathrm{~d}^{-1}\right)$ é a extração de água média dos dois compartimentos:

$$
\bar{S}_{e}=\frac{S_{e, A R}+S_{e, A G}}{2}=\frac{\Delta \theta_{A R}+\Delta \theta_{A G}}{2 \Delta t}
$$

Nessa equação, $S_{e, A R}$ e $S_{e, A G}$ são os valores de extração de água obtidos experimentalmente dos compartimentos $A R$ e $A G$, respectivamente, e $t(\mathrm{~d})$ é o tempo.

A qualidade da estimativa do modelo foi analisada pelos indicadores estatísticos erro absoluto médio (EAM), raiz do erro quadrático médio (REQM) e coeficiente de eficiência $E$ (Nash \& Sutcliffe, 1970) e pelo índice de concordância $d$ (Willmott, 1981):

$$
\begin{gathered}
E A M=\frac{\sum_{i=1}^{N}\left|S_{e, i}-S_{m, i}\right|}{N} \\
R E Q M=\sqrt{\frac{\sum_{i=1}^{N}\left(S_{e, i}-S_{m, i}\right)^{2}}{N}} \\
E=1-\frac{\sum_{i=1}^{N}\left(S_{e, i}-S_{m, i}\right)^{2}}{\sum_{i=1}^{N}\left(S_{e, i}-\bar{S}_{e}\right)^{2}} \\
d=1-\frac{\sum_{i=1}^{N}\left(S_{e, i}-S_{m, i}\right)^{2}}{\sum_{i=1}^{N}\left(\left|S_{m, i}-\bar{S}_{e}\right|+\left|S_{e, i}-\bar{S}_{e}\right|\right)^{2}}
\end{gathered}
$$

em que $S_{m}$ é a extração de água calculada pelo modelo (equação 10) e $S_{e}$ o valor da extração obtido experimentalmente para as $N$ observações nos dois compartimentos juntos.

\section{RESULTADOS E DISCUSSÃO}

\section{Sistema radicular}

Os valores de matéria seca, comprimento radicular $L$, densidade radicular $R$ e meia distância média entre raízes $r_{m}$ obtidos nos compartimentos com os solos AG e AR estão apresentados no quadro 2. Verifica-se densidade radicular e massa radicular maiores no solo AR do que no AG. Um levantamento feito por Willigen \& van Noordwijk (1987) identificou valores predominantemente da ordem de $5.10^{3}$ a $5.10^{4} \mathrm{~m} \mathrm{~m}^{-3}$. Assim, os valores aqui reportados representam uma densidade radicular abaixo da normalmente encontrada em culturas agrícolas. 
Quadro 2. Matéria seca de raiz determinada nos compartimentos dos lisímetros, comprimento de raiz $(L)$, densidade radicular $(R)$, distância média entre as raízes $\left(r_{m}\right)$ e parâmetro $\rho$ para os solos arenoso (AR) e argiloso (AG)

\begin{tabular}{|c|c|c|c|c|c|c|}
\hline Lisímetro & Solo & Matéria seca & $L$ & $\boldsymbol{R}$ & $r_{m}$ & $\rho$ \\
\hline & & $\mathrm{g}$ & $\mathrm{m}$ & $\mathrm{m} \mathrm{m}^{-3}$ & $\mathrm{~m}$ & $\mathrm{~m}^{-2}$ \\
\hline 1 & $\begin{array}{l}\mathrm{AR} \\
\mathrm{AG}\end{array}$ & $\begin{array}{l}6,230 \\
2,379\end{array}$ & $\begin{array}{l}44,07 \\
16,83\end{array}$ & $\begin{array}{r}1588 \\
606\end{array}$ & $\begin{array}{l}0,014 \\
0,023\end{array}$ & $\begin{array}{l}3235 \\
1069\end{array}$ \\
\hline 2 & $\begin{array}{l}\mathrm{AR} \\
\mathrm{AG}\end{array}$ & $\begin{array}{l}8,606 \\
4,049\end{array}$ & $\begin{array}{l}60,88 \\
28,64\end{array}$ & $\begin{array}{l}2194 \\
1032\end{array}$ & $\begin{array}{l}0,012 \\
0,018\end{array}$ & $\begin{array}{l}4716 \\
1966\end{array}$ \\
\hline 3 & $\begin{array}{l}\text { AR } \\
\text { AG }\end{array}$ & $\begin{array}{l}7,541 \\
4,612\end{array}$ & $\begin{array}{l}53,34 \\
32,62\end{array}$ & $\begin{array}{l}1922 \\
1175\end{array}$ & $\begin{array}{l}0,013 \\
0,016\end{array}$ & $\begin{array}{l}4041 \\
2284\end{array}$ \\
\hline 4 & $\begin{array}{l}\mathrm{AR} \\
\mathrm{AG}\end{array}$ & $\begin{array}{l}8,236 \\
4,245\end{array}$ & $\begin{array}{l}58,26 \\
30,03\end{array}$ & $\begin{array}{l}2099 \\
1082\end{array}$ & $\begin{array}{l}0,012 \\
0,017\end{array}$ & $\begin{array}{l}4479 \\
2076\end{array}$ \\
\hline
\end{tabular}

\section{Propriedades hidráulicas}

As curvas de retenção dos dois solos estão representadas na figura 3 , e os respectivos parâmetros de ajuste da equação 4 de van Genuchten (1980) encontram-se no quadro 3 . Os resultados experimentais para a relação entre a condutividade hidráulica $K$ e o potencial matricial $h$ e o ajuste de acordo com a equação 5 estão representados na

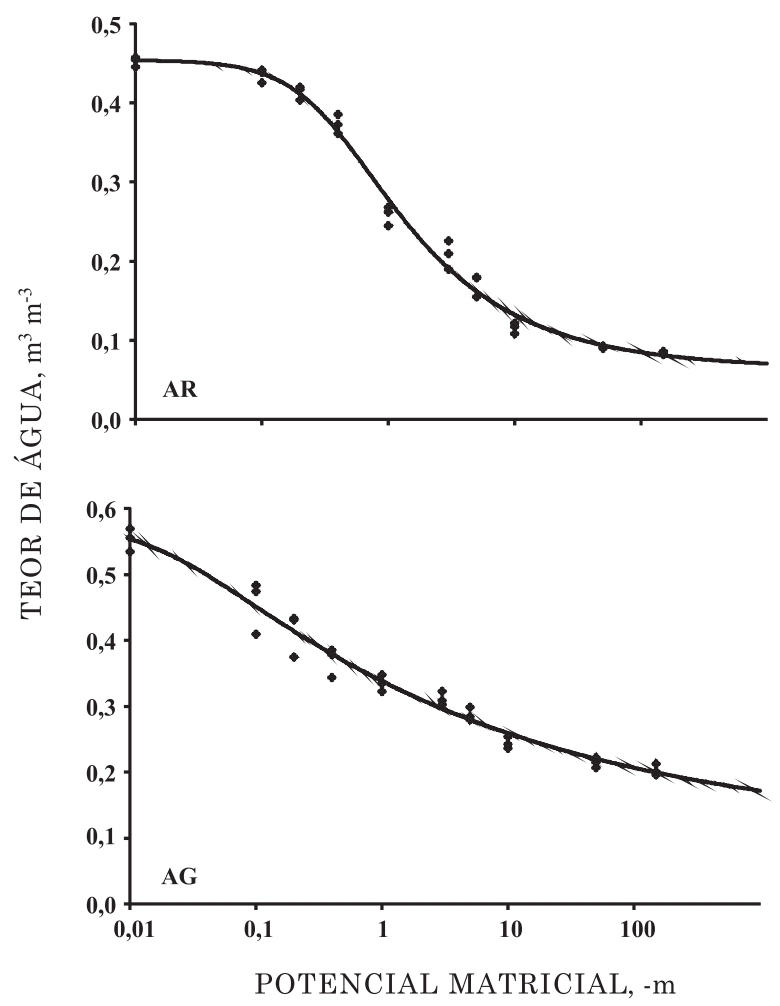

Figura 3. Teor de água em função do potencial matricial nos solos arenoso (AR) e argiloso (AG), sendo os dados obtidos experimentalmente representados por pontos e a equação de van Genuchten (1980) ajustada representada pelas linhas.
Quadro 3. Parâmetros de ajuste da equação de van Genuchten (1980) e da relação(1) entre a condutividade hidráulica $(K)$ e o potencial matricial (h) para os solos arenoso (AR) e argiloso (AG) utilizados no experimento

\begin{tabular}{lcc}
\hline Solo & AR & AG \\
\hline$\theta_{r}$ & 0,0646 & 0,1033 \\
$\theta_{s}$ & 0,4542 & 0,5809 \\
$\alpha\left(\mathrm{m}^{-1}\right)$ & 27,457 & 52,324 \\
$n$ & 15,268 & 11,792 \\
$\mathrm{R}^{2}$ & 0,99 & 0,97 \\
$K_{s}\left(\mathrm{~m} \mathrm{~d}^{-1}\right)$ & 0,06454 & 0,06034 \\
$\left|h_{b}\right|(\mathrm{m})$ & 0,4211 & 10,000 \\
$b$ & 29,995 & 43,692 \\
\hline \multirow{2}{*}{$K=K_{s}$ para $|h| \leq\left|h_{b}\right| K=K_{s}\left(\frac{\left|h_{b}\right|}{|h|}\right)^{b}$ e para $|h|>\left|h_{b}\right|}$.
\end{tabular}

figura 4. Os parâmetros da equação 5 estão no quadro 3 . Observa-se que os valores de $K_{s}$ foram da mesma ordem de grandeza para os dois solos e, como esperado, o potencial matricial de entrada de $\operatorname{ar}\left(h_{b}\right)$ foi menor no solo AR do que no AG.

Utilizando os parâmetros obtidos para as equações 4 e 5 , podem-se determinar as relações entre o potencial de fluxo matricial $M$ e os valores de $h$ ou $\theta$ (Figura 5). Com o auxílio dessas relações, a densidade de fluxo entre dois pontos no solo pode ser determinada pelo gradiente de $M$ (equação 8), em função dos valores respectivos do potencial matricial ou do teor de água. Esse é o princípio empregado no modelo para o cálculo da absorção de água pelo sistema radicular.

\section{Observações experimentais nos lisímetros}

A figura 6 mostra o teor de água e o potencial de fluxo matricial nos lisímetros em função do tempo, durante os 46 dias do período de observações experimentais. A inclinação da linha $\theta-t(d \theta / d t)$ representa a taxa de extração de água. Observam-se claramente as oscilações do teor de água devido às 

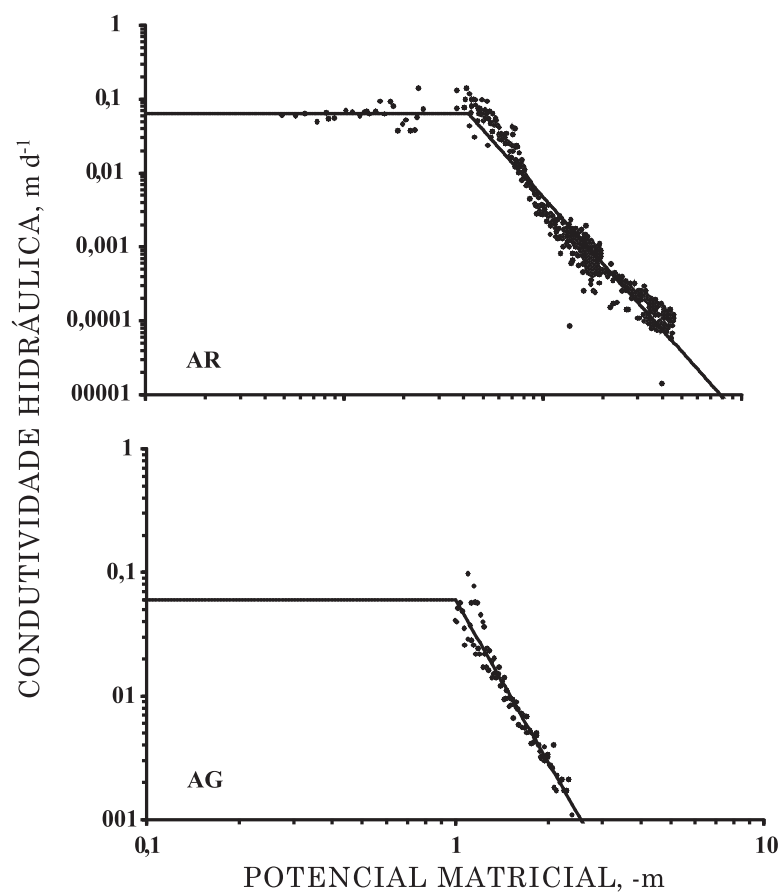

Figura 4. Condutividade hidráulica em função do potencial matricial nos solos arenoso (AR) e argiloso (AG). Pontos representam os valores observados, e a linha, o ajuste da equação $K=K$, para $|h| \leq\left|h_{b}\right|$ e $K=K_{s}\left(\frac{\left|h_{b}\right|}{|h|}\right)^{b}$ para $|h|>\left|h_{b}\right|$.

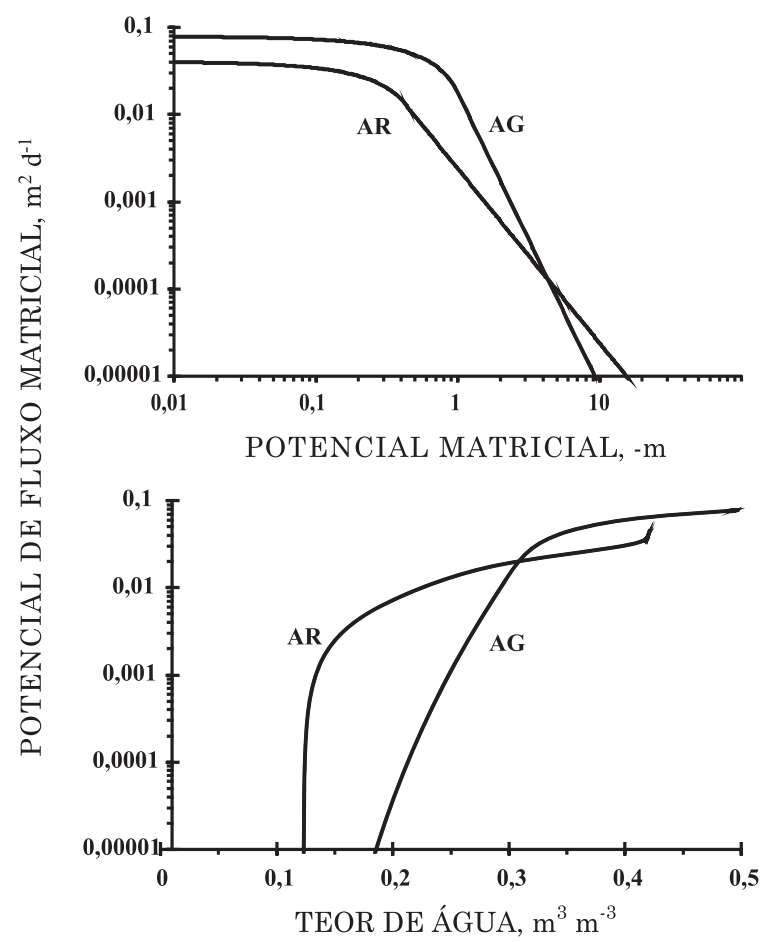

Figura 5. Potencial de fluxo matricial em função do potencial matricial e do teor de água nos solos arenoso (AR) e argiloso (AG) utilizados no experimento. extrações e reposições. Em todos os lisímetros foi possível observar que a extração de água se intensificou no compartimento onde ocorreu o potencial de fluxo matricial maior. Os valores de $M$ são indicadores da facilidade que o sistema radicular tem para extrair a água do solo: quanto maior o $m$, mais fácil a extração do respectivo compartimento (equação 10).

Verifica-se que, nos dias antes da primeira irrigação, a extração da água nos dois solos (AR e AG) ocorreu com intensidade parecida. Até o início da fase III (em 6 de junho), o valor de $M$ foi sempre maior no solo AR, irrigado na fase I. Portanto, pela equação 10, considerando $M_{0}$ e $\rho$ da mesma ordem em ambos os compartimentos, esperava-se uma extração de água mais intensa do solo AR, o que de fato foi observado durante todo esse período.

Na fase II percebe-se maior extração do solo AR enquanto este apresentou maior valor de $M$. Nos dias 29 e 30 de maio houve redução momentânea da extração, correlacionada com alguns dias com menores temperaturas e maiores valores de umidade relativa observados. A partir de 4 de junho os valores de $M$ dos dois lados ficaram parecidos, e a extração de ambos intensificou-se de modo geral. Na fase III (a partir de 6 de junho), quando o solo AG recebeu irrigação, a extração do AR cessou. Assim que o solo AG foi irrigado, a extração do solo AR terminou, demonstrando uma resposta muito rápida da planta em relação a mudanças no ambiente radicular.

Observando detalhadamente o teor de água do solo AR quando não irrigado, no período a partir de 6 de junho, verifica-se aumento de $\theta$, que pode ser atribuído à transferência de água do lado de maior potencial matricial ao lado de menor potencial matricial por meio das raízes da própria planta. Esse fenômeno, denominado de hydraulic lift, tem sido observado por diversos autores (Dirksen \& Raats, 1985; Richards \& Caldwell, 1987; Leffler et al., 2005). Em 15 de junho, observa-se, em todos os lisímetros, redução do teor de água no solo AR. Possivelmente houve, nesse dia, maior dificuldade de extração do solo AG em razão do término das irrigações. Com exceção do lisímetro 1 , os valores de $M$ nesse dia se igualam nos dois solos, o que reforça essa hipótese. Não está claro, no entanto, por que a extração ocorre apenas do solo AG nos dias subsequentes.

\section{Avaliação do desempenho do modelo}

Os valores de extração observados nos lisímetros, quando comparados aos estimados pela equação 10, revelaram ausência de correlação. Essa falta de correlação pode ser atribuída a diversos fatores, entre eles a dificuldade na determinação do valor do parâmetro $\rho$ e o fato de o modelo considerar que o sistema radicular é homogêneo, está em contato perfeito com o solo e com atividade igual em todas as raízes. A distribuição real do sistema radicular é desordenada, devido ao crescimento preferencial das raízes em rachaduras e macroporos do solo, 

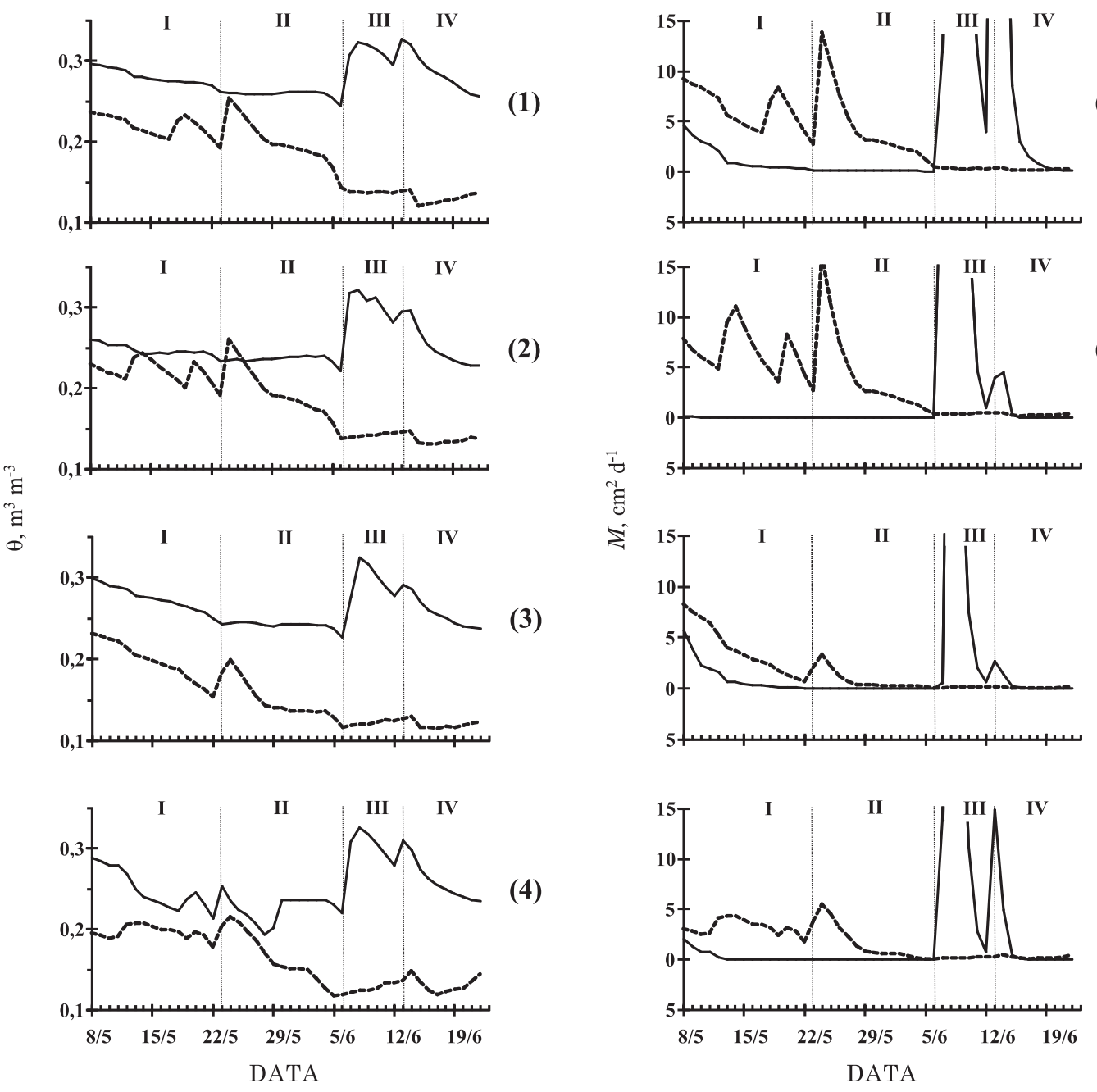

(1)

(2)

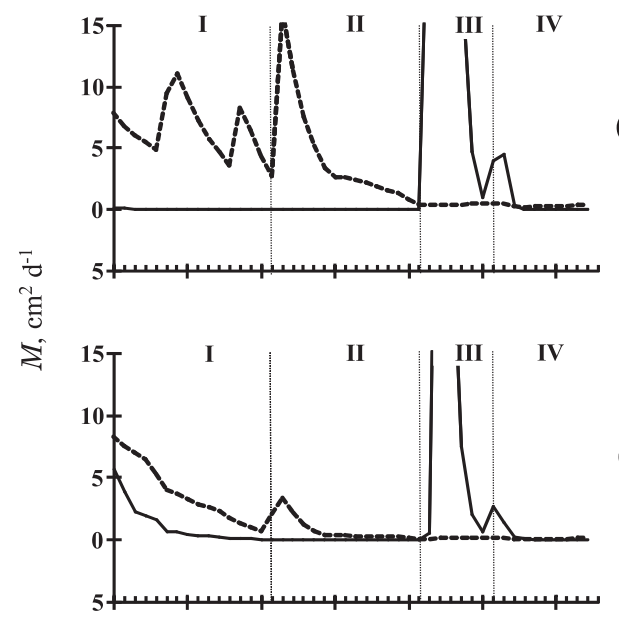

(2)

(3)

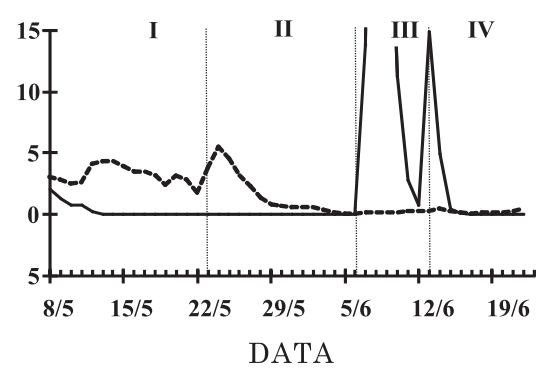

(4)

Figura 6. Teor de água $(\theta)$ e potencial de fluxo matricial $(M)$ durante o período experimental em 2008 nos lisímetros 1, 2, 3 e 4. As linhas contínua e pontilhada representam o solo AG e AR, respectivamente.

ocasionando o acúmulo de raízes em determinado local (Tardieu \& Manichon, 1986; Passioura, 1988). Essa desuniformidade resulta em maiores distâncias médias entre as raízes, comparativamente à distribuição homogênea considerada pelo modelo, sobretudo quando há menor eficiência na extração de água. Quanto à atividade das raízes, nem todas elas contribuem igualmente na extração de água do solo (Tofinga \& Snaydon, 1992), tendo as mais velhas e lignificadas uma atividade reduzida quando comparadas às mais novas. O contato da raiz com o solo também nem sempre é perfeito, podendo gerar uma resistência adicional na interface solo-raiz (Faiz \& Weatherley, 1982; Passioura, 1988). Para a correção empírica desse conjunto de fatores, um parâmetro $f$ foi incluído na equação 10, tornando-a:

$$
S=f \rho\left(\bar{M}-M_{0}\right)
$$

O valor desse parâmetro foi determinado minimizando-se o erro absoluto médio (EAM), de acordo com a equação 14. Para isso, utilizou-se um processo iterativo para todos os lisímetros e compartimentos simultaneamente, encontrando-se valores de 0,01506 e 0,003713 para os compartimentos com solo AR e AG, respectivamente. Valores da mesma ordem de grandeza foram relatados por Herkelrath et al. (1977), que realizaram uma correção semelhante. O desempenho do modelo foi avaliado depois de utilizados esses fatores de correção.

Os indicadores estatísticos da análise do desempenho do modelo são apresentados no quadro 4. A interpretação desses indicadores é tratada extensivamente por Legates \& Mccabe (1999). Segundo esses autores, não existe um indicador estatístico único que possa avaliar o desempenho de modelos. Vários indicadores, juntamente com uma análise gráfica, devem ser utilizados. Indicadores absolutos, como o EAM e o REQM, têm a mesma unidade da grandeza avaliada e são, portanto, de mais fácil interpretação que os indicadores adimensionais, como $R^{2}, E$ e $d$.

Em relação ao $E$, quando este apresenta valor negativo, significa que o modelo prediz os valores observados 
com menor precisão do que o valor médio observado. Isso é verificado para todos os lisímetros (Quadro 4). Os valores de $d$ também são baixos, ao redor de 0,5 , enquanto os erros EAM e REQM apresentam valores da ordem de 0,005 a $0,1 \mathrm{~m}^{3} \mathrm{~m}^{-3} \mathrm{~d}^{-1}$, o que significa que o desempenho do modelo não foi bom.

A fim de verificar se esse desempenho foi ruim para todos os valores experimentais, ou se valores discrepantes influenciariam negativamente os indicadores, foram calculados também os indicadores para o conjunto de dados desconsiderando-se os $20 \%$ dos valores com o maior erro absoluto (Quadro 4). Os valores desconsiderados correspondem, quase na sua totalidade, a predições de extração de água do compartimento irrigado e liberação no compartimento não irrigado. Os indicadores melhoraram significativamente após essa remoção de valores, o que significa que valores discrepantes influenciaram bastante o resultado final. O conjunto de dados reduzido apresenta indicadores aceitáveis: valores de erros em torno de 0,002 a 0,005 $\mathrm{m}^{3} \mathrm{~m}^{-3} \mathrm{~d}^{-1}$; valores de $E$ todos positivos e, exceto no lisímetro 4, altos (ao redor de 0,5$)$; e valores de $d$ altos, da ordem de 0,8. Assim, o modelo descreveu bem 80 \% das observações com a utilização dos respectivos valores de $f$.

O desempenho do modelo não foi igual para os quatro lisímetros (unidades experimentais), como indicam os valores dos indicadores (Quadro 4); os resultados para o lisímetro 4 foram os piores, como pode ser observado também na figura 7. O desempenho também foi diferente entre os solos: para o solo AR, os pontos observados se aproximaram mais dos valores

Quadro 4. Índices estatísticos da comparação entre valores da extração da água do solo observados e determinados pelo modelo para o conjunto de dados completo e excluindo-se os $20 \%$ dos valores de maior erro quadrado

\begin{tabular}{|c|c|c|c|c|}
\hline & \multicolumn{4}{|c|}{ Lisímetro } \\
\hline & 1 & 2 & 3 & 4 \\
\hline \multicolumn{5}{|c|}{ Conjunto de dados completo } \\
\hline$E A M\left(\mathrm{~m}^{3} \mathrm{~m}^{-3} \mathrm{~d}^{-1}\right)$ & 0,00445 & 0,00545 & 0,00441 & 0,00690 \\
\hline$R E Q M\left(\mathrm{~m}^{3} \mathrm{~m}^{-3} \mathrm{~d}^{-1}\right)$ & 0,00670 & 0,00798 & 0,00806 & 0,01037 \\
\hline$E$ & $-1,412$ & $-0,640$ & $-1,788$ & $-1,410$ \\
\hline$D$ & 0,539 & 0,632 & 0,494 & 0,476 \\
\hline \multicolumn{5}{|c|}{ Excluindo $20 \%$ dos desvios maiores } \\
\hline$E A M\left(\mathrm{~m}^{3} \mathrm{~m}^{-3} \mathrm{~d}^{-1}\right)$ & 0,00253 & 0,00309 & 0,00224 & 0,00403 \\
\hline$R E Q M\left(\mathrm{~m}^{3} \mathrm{~m}^{-3} \mathrm{~d}^{-1}\right)$ & 0,00306 & 0,00378 & 0,00274 & 0,00499 \\
\hline$E$ & 0,416 & 0,562 & 0,453 & 0,208 \\
\hline$d$ & 0,815 & 0,883 & 0,836 & 0,762 \\
\hline
\end{tabular}
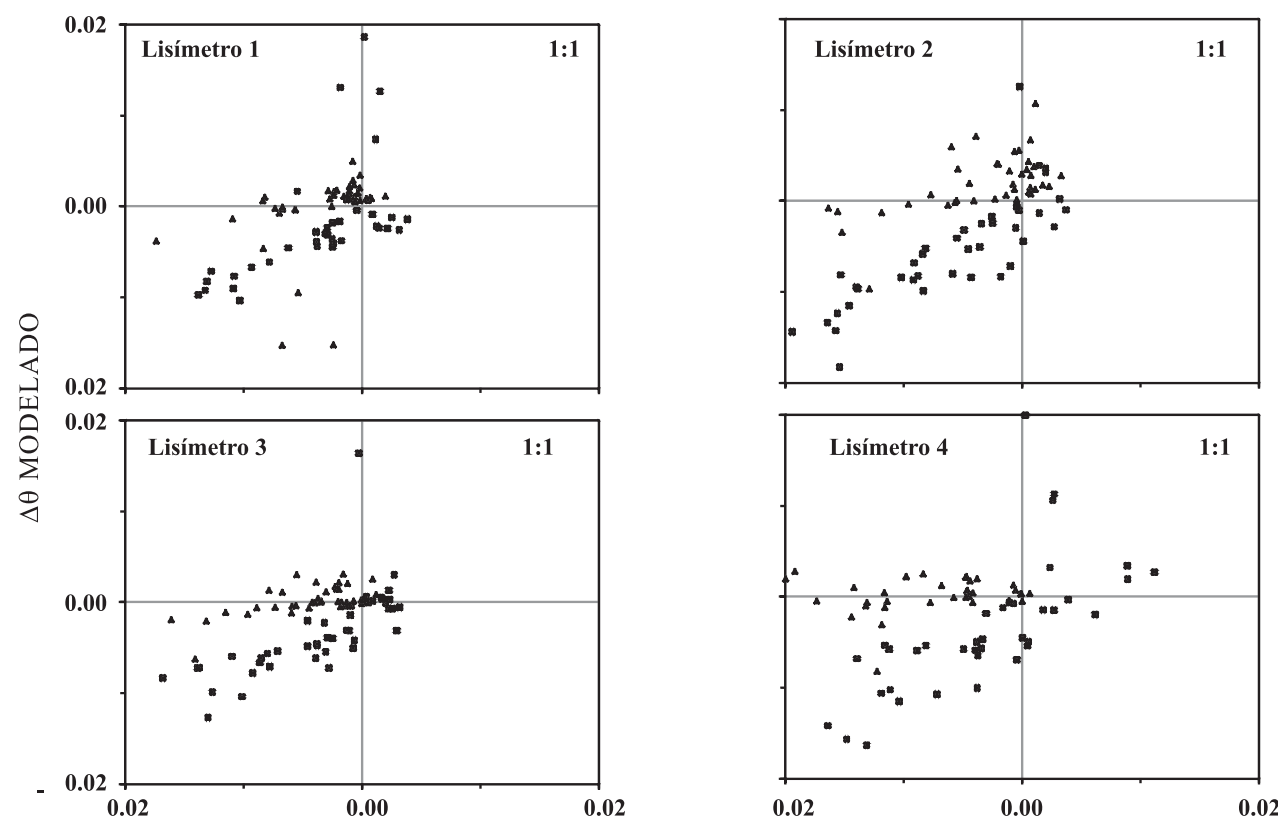

$\triangle \theta$ OBSERVADO

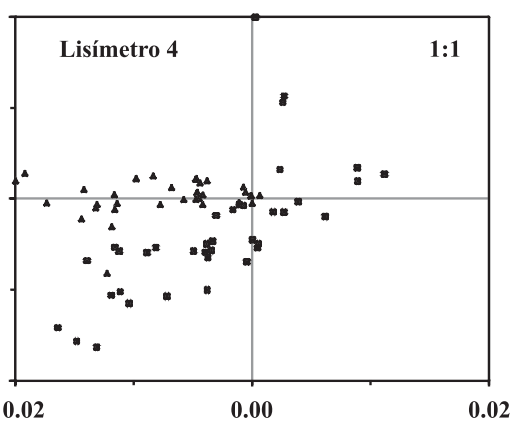

Figura 7. Valores de $\Delta \theta$ observados e obtidos pelo modelo nos lisímetros 1, 2, 3 e 4. Quadrados e triângulos representam observações nos solos arenoso (AR) e argiloso (AG), respectivamente. 
preditos, com melhor aproximação nos lisímetros 2 e 3 e maior dispersão dos pontos no lisímetro 4. Para o solo AG, os valores mostraram-se mais distantes da reta $1: 1$.

Considerar que o valor de $M_{0}$ é igual nos dois solos AR e AG pode, também, ter sido uma fonte de erro de estimativa pelo modelo. Na extrapolação de modelos microscópicos da extração radicular, é comum supor que os valores de potencial matricial são semelhantes em toda a superfície radicular (Javaux et al., 2008; Jong van Lier et al., 2008), mas diferenças na relação $M h$ (Figura 5) podem ter levado a desvios.

$\mathrm{Na}$ figura 7 observa-se, ainda, a estimativa de valores positivos de $\Delta \theta$ pelo modelo com maior frequência e intensidade do que esses valores foram observados. Em outras palavras, o modelo prevê a liberação de água das raízes ao solo mais frequentemente do que ela realmente ocorre. A inclusão da resistência hidráulica do sistema radicular, sugerida por Javaux et al. (2008) e atualmente desconsiderada pelo modelo, deve melhorar seu desempenho nesse sentido.

\section{CONCLUSÕES}

1. O modelo utilizado simula a liberação de água ao solo mais frequente e mais intensamente do que ela ocorreu no experimento. Esse fato deve-se provavelmente à existência de resistência interna do sistema radicular, não contabilizada pelo modelo.

2. Após a eliminação de $20 \%$ de valores discrepantes, que representaram, principalmente, as ocasiões de liberação de água pelo sistema radicular no comportamento de menor potencial de fluxo matricial, os indicadores estatísticos demonstram que o modelo com fator de correção descreve satisfatoriamente as observações.

\section{AGRADECIMENTOS}

À Fapesp, pelas bolsas de estudo; e à aluna de doutorado Angelica Durigon e ao técnico de laboratório Gilmar Batista Grigolon, pelo auxílio nas análises dos parâmetros hidráulicos dos solos.

\section{LITERATURA CITADA}

AMATO, M. \& PARDO, A. Root length and biomass losses during sample preparation with different screen mesh sizes. Plant Soil, 161:299-303, 1994.

CLAUSNITZER, V. \& HOPMANS, J.W. Simultaneous modeling of transient three-dimensional root growth and soil water flow. Plant Soil, 164:299-314, 1994.
COWAN, I.R. Transport of water in the soil-plant-atmosphere system. J. Appl. Ecol., 2:221 239, 1965.

CRAINE, J.M. \& LEE, W.G. Covariation in leaf and root traits for native and non-native grasses along an altitudinal gradient in New Zealand. Ecophysiology, 134:471-478, 2003.

DIRKSEN, C. \& RAATS, P.A.C. Water uptake and release by alfalfa roots. Agron. J., 77:621-626, 1985.

DOORENBOS J. \& KASSAM, A.H. Yield response to water. Rome, Food and Agricultural Organization of the United Nations, 1986. (FAO Irrigation and Drainage Paper, 33)

EMPRESA BRASILEIRA DE PESQUISA AGROPECUÁRIA . EMBRAPA. Sistema brasileiro de classificação de solos. Brasília, Centro Nacional de Pesquisa em Solos, 1999. $412 \mathrm{p}$.

ESPELETA, J.F.; EISSENSTAT, D.M. \& GRAHAM, J.H. Citrus root responses to localized drying soil: A new approach to studying mycorrhizal effects on the roots of mature trees. Plant Soil, 206:1-10, 2004.

FAIZ, S.M.A. \& WEATHERLEY, P.E. Root contraction in transpiring plants. New Phytol., 92:333-343, 1982.

FEDDES, R.A.; KOWALIK, P.J. \& ZARADNY, H. Simulation of field water use and crop yield.. Wageningen, PUDOC, 1978. (Simulation Monograph Series)

GARDNER, W.R. Some steady-state solutions of the unsaturated moisture flow equation with application to evaporation from a water table. Soil Sci., 85:228-232, 1958.

GARDNER, W.R. Dynamic aspects of water availability to plants. Soil Sci., 89:63-67, 1960.

GARDNER, W.R. Relation of root distribution to water uptake and availability. Agron. J., 56:41-45, 1964.

HERKELRATH, W.N.; MILLER, E.E. \& GARDNER, W.R. Water uptake by plants: II. The root contact model. Soil Sci. Soc. Am. J., 41:1039-1043, 1977.

HUSSAIN, A.; BLACK, C.R.; TAYLOR, I.B. \& ROBERTS, J.A. Soil compaction. A role for ethylene in regulating leaf expansion and shoot growth in tomato? Plant Physiol., 121:1227-1237, 1999

JAVAUX, M.; SCHRÖDER, T.; VANDERBORGHT, J.; \& VEREECKEN, H. Use of a three-dimensional detailed modeling approach for predicting root water uptake. Vadose Zone J., 7:1079-1088, 2008.

JONG van LIER, Q.; METSELAAR, K. \& van DAM, J.C. Root water extraction and limiting soil hydraulic conditions estimated by numerical simulation. Vadose Zone J., 5:1264 $1277,2006$.

JONG van LIER, Q.; METSELAAR, K.; van DAM, J.C.; JONG, R. \& DUIJNISVELD, W.H.M. Macroscopic root water uptake distribution using a matric flux potential approach. Vadose Zone J., 7:1065-1078, 2008.

KOSOLA, K.R. \& EISSENSTAT, D.M. The fate of surface roots of citrus seedlings in dry soil. J. Exper. Bot., 45:1639$1645,1994$. 
KOZAK, J.A.; AHUJA, L.M. \& GREEN, T.R. Scaling and estimation of evaporation and transpiration of water across soil textures. Vadose Zone J., 4:418-427, 2005.

KROES, J.G. \& van DAM, J.C. Reference manual SWAP version 3.0.3. Alterra Rep. 773. Wageningen, Research Institute for the Green World, 2003. 211p.

LAI, C.T. \& KATUL, G. The dynamic role of root-water uptake in coupling potential to actual transpiration. Adv. Water Res., 23:427-439, 2000.

LASCANO, R.J. \& van BAVEL, H.M. Root water uptake and soil water distribution: Test of an availability concept. Soil Sci. Soc. Am. J. 48:233-237, 1986.

LEGATES, D.R. \& MCCABE, G.J. Evaluating the use of "goodness-of-fit" measures in hydrologic and hydroclimatic model validation. Water Res. Res., 35:233-241, 1999.

LEFFLER, A.J.; PEEK, M.S.; RYEL, R.J.; IVANS, C.Y. \& CALDWELL, M.M. Hydraulic redistribution through the root systems of senesced plants. Ecology, 86:633-642, 2005.

LI, K.Y.; DE JONG, R \& BOISVERT, J.B. Comparison of root water uptake models. In: STOTT, D.E.; MOHTAR, R.H. \& STEINHARDT, G.C., eds. SUSTAINING THE GLOBAL FARM: SELECTED FROM THE 10TH INTERNATIONAL SOIL CONSERVATION ORGANIZATION MEETING, West Lafayette, 1999. Papers... West Lafayette, Purdue University, 2001a. p.1112-1117.

LI, K.Y.; DE JONG, R. \& BOISVERT, J.B. An exponential root-water-uptake model with water stress compensation. J. Hydrol., 252:189-204, 2001b.

MOLZ, F.J. Soil-plant water transport. Water Res. Res., 17:1245-1260, 1981.

MOLZ, F.J. \& REMSON, I. Extraction term models of soil moisture use by transpiring plants. Water Res. Res., 6:1346-1356, 1970.

NASH, J.E. \& SUTCLIFFE, J.V. River flow forecasting through conceptual models, I, A discussion of principles. J. Hydrol., 10:282-290, 1970.

PASSIOURA, J.B. Water transport in and to roots. Ann. Rev. Plant Physiol., 39:245-265, 1988.

RAATS, P.A.C. Steady infiltration from line sources and furrows. Soil Sci. Soc. Am. Proc., 34:709-714, 1970.

RAIJ, B.van; CANTARELLA, H.; QUAGGIO, J.A. \& FURLANI, A.M.C., eds. Recomendações de adubação e calagem para o Estado de São Paulo. 2.ed. Campinas, Instituto Agronômico de Campinas, 1997. 285p. (IAC. Boletim Técnico, 100)
RICHARDS, J.H. \& CALDWELL, M.M. Hydraulic lift: Substantial nocturnal water transport between soil layers by Artemisia tridentata roots. Oecologia, 73:486-489, 1987.

RITCHIE, J.T. A user-orientated model of the soil water balance in wheat: Models in wheat agronomy. In: DAY, W. \& ATKIN, R.K., eds. Wheat growth and modeling. New York: Plenum Press, 1985. p.293-305.

TARDIEU, F. \& MANICHON, H. Caractérization en tant que capteur d'eau de l'enracinement du mais en parcelle cultivée. 2 . Une méthode d'étude de la répartition verticale et horizontale des racines. Agronomie, 6:415-425, 1986.

TOFINGA, M.P. \& SNAYDON, R.W. The root activity of cereals and peas when grown in pure stands and mixtures. Plant Soil, 142:281-285, 1992.

van GENUCHTEN, M.T. A closed-form equation for predicting the hydraulic conductivity of unsaturated soils. Soil Sci. Soc. Am. J., 44:892-897, 1980.

van DAM, J.C.; STRICKER, J.N.M. \& DROOGERS, P. Inverse method to determine soil hydraulic functions from Multistep outflow experiments. Soil Sci. Soc. Am. J., 58:647652, 1994.

van den BERG, M. \& DRIESSEN, P.M. Water uptake in crop growth models for land use systems analysis: I. A review of approaches and their pedigrees. Agric. Ecosyst. Environ., 92:21-36, 2002.

van NOORDWIJK, M. \& BROUWER, G. Review of quantitative root length data in agriculture. In: MCMICHAEL, B.L. \& PERSSON, H., eds. Plant roots and their environment. New York, Elsevier, 1991. p.515525 .

WILLIAMS, J.R. The EPIC model. In: SINGH, V.P., ed. Computer models of watershed hydrology. Littleton, Water Resources Publ., 1995. p.909-1000.

WILLIGEN, P. \& van NOORDWIJK, M. Roots, plant production and nutrient use efficiency. Wageningen, Agricultural University Wageningen, 1987. (Tese de Doutorado)

WILLMOTT, C.J. On the evaluation of models. Phys. Geogr., 2:184-194, 1981.

WIND, G.P. Capillary conductivity data estimated by a simple method. In: RIJTEMA, P.E. \& WASSINK, H., eds. WATER IN THE UNSATURATED ZONE SYMPOSIUM., Wageningen, 1966. Proceedings...Gentbrugge, International Association Scientific Hydrology, 1968. v.1. 